\title{
Mitochondrial Transfer through Pericardium Contributes to Functional Recovery in Ischemic Cardiomyopathy
}

Daisuke MORI

Osaka University

Shigeru MIYAGAWA

Osaka University

Takuji KAWAMURA

Osaka University

Daisuke YOSHIOKA

Osaka University

Hiroki HATA

Osaka University

Takayoshi UENO

Osaka University

Koichi TODA

Osaka University

Toru KURATANI

Osaka University

Miwa 00TA

ROHTO Pharmaceutical Co., Ltd.

Kotoe KAWAI

ROHTO Pharmaceutical Co., Ltd.

Hayato KURATA

ROHTO Pharmaceutical Co., Ltd.

Hiroyuki NISHIDA

ROHTO Pharmaceutical Co., Ltd.

Akima HARADA

Osaka University

Toshihiko TOYOFUKU

Osaka University

Yoshiki SAWA ( $\nabla$ sawa-p@surg1.med.osaka-u.ac.jp )

Osaka University 


\section{Research Article}

Keywords: mesenchymal, transplantation, cardiomyocytes

Posted Date: November 24th, 2020

DOI: https://doi.org/10.21203/rs.3.rs-107042/v1

License: (c) (i) This work is licensed under a Creative Commons Attribution 4.0 International License. Read Full License 


\section{Abstract}

Although mesenchymal stem cell transplantation has been efficacious in the treatment of ischemic cardiomyopathy, the underlying mechanisms remain unclear. Herein, we investigated whether mitochondrial transfer could explain the success of cell therapy in ischemic cardiomyopathy. Mitochondrial transfer was examined in co-cultures of human adipose-derived mesenchymal stem cells and rat cardiomyocytes under hypoxic conditions. Functional recovery was monitored in a rat model of myocardial infarction following human adipose-derived mesenchymal stem cell transplantation. In vitro, we observed mitochondrial transfer, which required formation of cell-to-cell contacts and synergistically enhanced energy metabolism. Rat cexhibited mitochondrial transfer three days following human adiposederived mesenchymal stem cell transplantation to the ischemic heart surface post myocardial infarction. We detected donor mitochondrial DNA in the recipient myocardium concomitant with a significant improvement in cardiac function. In conclusion, mitochondrial transfer is vital for successful cell transplantation therapies and promotes improved treatment outcomes in ischemic cardiomyopathy.

\section{Introduction}

Ischemic heart disease is one of the leading cause of death worldwide. Various cell therapies have been applied to remediate acute myocardial infarction and ischemic cardiomyopathy. The modes of action of these therapies include angiogenesis, cardiomyocyte regeneration, protection from ischemia reperfusion injury, and activation of hibernating myocardium, mediated by cytokines, exosomes, and microRNAs secreted from transplanted cells. However, the mechanisms underlying these responses remain to be fully elucidated [1-4].

Recently, a phenomenon, termed mitochondrial transfer, has been described in animal models of acute lung injury following mesenchymal stem cell (MSC) transplantation. Mitochondrial transfer is thought to exert protective effects on recipient alveolar epithelial cells, thereby representing a potential cell therapy mode of action against heart failure [5-9]. Furthermore, bidirectional mitochondrial transfer has been observed in cardiomyocytes co-cultured with MSCs $[5,10,11]$. Cardiomyocytes receiving mitochondria show elevated expression of mitochondrial proteins, increased oxidative phosphorylation, and improved ATP production [12,13]. Meanwhile, in vivo, mitochondrial transfer is expected to confer resistance to cytotoxicity, exert anti-apoptotic effects, and increase ATP production in recipient cardiomyocytes, thus facilitating an overall improvement in myocardial function [14-16].

To the best of our knowledge, no previous study has evaluated whether mitochondrial transfer occurs in vivo following cell transplantation to the heart. Thus, the role of mitochondrial transfer in the success of cell transplantation and its effect on heart function remains unknown. In this study, we demonstrated mitochondrial transfer from transplanted cells to cardiomyocytes in vivo and proposed this as the mechanism underlying successful cell therapies for ischemic cardiomyopathy (Fig. 1).

\section{Results}


Three days following transplantation of hADSCs harboring pre-stained mitochondria, we detected stained mitochondria in the recipient rat heart and epicardium (Fig. 1A-D). Electron microscopy revealed tunnellike connections between hADSCs and cardiomyocytes (Fig. 1E-G). Laser microdissection was used to obtain heart samples for DNA sequencing (Fig. 1H). The mitochondrial genome fragment was PCRamplified, and the resultant amplicon length was consistent with those in previous reports (Fig. 1I) [21]. The genome sequence analysis revealed $99 \%$ homology with human mitochondrial NADH-ubiquinone oxidoreductase chain 6 (MT-ND6) (Fig. 1J).

\section{Mitochondrial transfer occurs within 24 h following transplantation}

For intravital imaging, we transfected hADSCs with CellLight to induce mitochondrial expression of red fluorescent protein (Fig. 2A-B and Supplementary Video S1A online). Upon transplantation, the graft formed a layer on the heart where hADSCs were equally distributed (Fig. 2C and Supplementary Video S1B online). Three to $4 \mathrm{~h}$ post transplantation, mitochondria from the hADSCs were detected in the myocardial layer of the recipient (Fig. 2D and Supplementary Video S1C online). Residual cardiomyocytes in the fibrotic area contained granules that were considered to represent the transferred mitochondria (Fig. 2E-F). When co-culture of hADSCs and cardiomyocytes was performed for $24 \mathrm{~h}$ under hypoxic conditions, the hADSCs were found to move around the cardiomyocytes in a fixed field of view (Fig. 3A-B and Supplementary Video S2A online). In the cardiomyocytes, the intensity of hADSC-derived mitochondrial luminescence significantly increased during the 24-h co-culture (Fig. $3 \mathrm{C}$ ). The fixed sample also exhibited granular hADSC-derived mitochondria in the rat cardiomyocytes (rCM) (Fig. 3D and Supplementary Video S2B online).

\section{hADSC co-culture synergistically enhances the OCR of cardiomyocytes}

The OCR of rCMs was synergistically enhanced by hADSC co-culture, being significantly higher than the sum of the OCRs of rCMs and hADSCs cultured independently. This effect was cancelled by the addition of a non-specific gap junction inhibitor aGA (Fig. 3E-F). We successfully obtained adult rCMs using a Langendorff circuit and captured the motion of individual rCMs (Fig. 3K-M). An additional 24-h culture with supernatant hADSCs had no effect on the contraction of rCMs (Fig. $3 \mathrm{~N}$ ).

\section{hADSC transplantation improves cardiac function in a dosedependent manner}

We monitored luciferin signals using an in vivo imaging system at least four weeks post transplantation. On the fourth week, the heart surface was observed directly (Fig. 4B-C). Histological analysis of these samples revealed surviving hADSCs on the surface or in the connective tissue between the heart and chest wall (Fig. 4D). The supernatant of cultured and grafted hADSCs contained various cytokines, including VEGF, HGF, interleukin (IL)-6, and IL-10. Among them, secretion of VEGF and IL-6 was stimulated by hypoxia (Fig. 4E). hADSC transplantation exhibited a therapeutic effect on cardiac function in the infarcted rats. The effect was temporary in low dose- and medium-dose groups, limited to the early phase 
following transplantation. In the max-dose group, improvement was greater than that in the rest at weeks 2 - 6. However, at week 8, cardiac function improved in both the low-dose and medium-dose groups equally. No effect against cardiac remodeling was observed, as indicated by left ventricular (LV) enddiastolic diameter (LVDd) and LV posterior wall thickness; however, the LV end-systolic diameter (LVDs) tended to become smaller with increasing dose (Fig. 4F and Supplementary Fig. S1 online). Although the addition of aGA to hADSCs blocked the improvement of cardiac function three days post transplantation, it did not affect the long-term improvement (Fig. 4G). The optimal transplantation time was found to be 1 -2 weeks following the onset of myocardial infarction (Supplementary Fig. S2A-J online).

\section{hADSC transplantation increases intramyocardial mitochondrial DNA and ATP}

No difference was observed in fibrotic area across the groups during the first two weeks following implantation. However, a difference emerged after eight weeks. Additionally, semiquantitative assessment revealed less interstitial fibrosis in the hADSC-treated group compared to the control group. rCM diameter was significantly smaller in the high-dose and maximum dose groups, and the highest capillary densities and lowest collagen accumulation were observed in the medium-dose group (Fig. 5AB). Mitochondrial number and cristae structure were relatively normal compared to that in the untreated group, as indicated by electron microscopy. Other organelles, such as the endoplasmic reticulum, myofibers, Z-disk, and intercalated disks, were morphologically preserved (Fig. 5C). Following treatment, the amount of rat mitochondrial DNA in the myocardium increased with time. However, mitochondrial DNA from the infarct area exhibited a decrease by day 56 post treatment (Fig. 5D). Donor-derived mitochondrial genes were not sufficiently amplified for detection. One day after transplantation, cytosolic ATP levels in the hADSC-treated group were approximately ten-fold higher than those in the control group (Fig. 5E).

\section{Discussion}

Following transplantation of hADSCs onto the ischemic heart surface, donor-derived mitochondrial DNA was detected in the DNA extracted from cardiomyocytes, providing the first evidence of mitochondrial transfer from ADSCs to the ischemic myocardium in vivo. This mitochondrial transfer occurred shortly following transplantation, as evidenced by histological evaluation and intravital imaging, suggesting an influence on early improvement of cardiac function. Furthermore, ATP concentration in the intramyocardial infarct area increased $24 \mathrm{~h}$ post transplantation and stabilized after a week. Previous reports have suggested retention of these mitochondria for approximately 1 week [28], that is consistent with our findings. However, direct histological identification of mitochondria over time was challenging. Although the detailed mechanisms underlying mitochondrial transfer have not been established, our data confirm cell-cell communication and indicate transfer of mitochondria and other organelles between cells [29]. Specifically, when cardiomyocytes were exposed to hADSC supernatant, no immediate contraction-enhancement effect was observed on cardiomyocytes, suggesting that a direct cell-cell communication is necessary for mitochondrial transfer to occur between hADSCs and cardiomyocytes [30]. In vitro experiments using a co-culture system demonstrated that ADSCs interact with and transfer 
mitochondria to cardiomyocytes, thereby improving their metabolic capacity, as measured using OCR. Moreover, addition of a connexin blocker to this in vitro co-culture system prevented the OCR enhancement effect of mitochondrial transfer and reduced the ability of mitochondrial transfer to improve cardiac function in vivo, suggesting the involvement of connexin in cell-cell communication underlying mitochondrial transfer. However, the survival period of transferred mitochondria, the signaling pathway leading to mitochondrial transfer, and the detailed mechanisms of recipient cell activation remain to be elucidated.

Ischemic diseases are often ameliorated by angiogenesis; however, this can lead to the dysfunction of myocardial and cardiac cells. Reactivation of these cells can result in long-term improvement of cardiac function. Although the transplanted cells survived on the surface of the heart for approximately four weeks, the transferred mitochondria could not be identified in heart specimens thereafter. Rather, the transferred mitochondria either disappeared within a few days following transplantation or were present in very low numbers. This suggests that hADSC treatment improves the pathophysiology of ischemic cardiomyopathy, including mitochondrial abnormalities, by providing a short-term supply of relatively healthy mitochondria and normalizing the long-term morphology and quantity of mitochondria in the recipient myocardium [14].

Regarding the pathophysiology of mitochondrial cardiomyopathy, mitochondrial protein abnormalities can cause chronic structural abnormalities in heart tissue. Thus, if transferred mitochondria activate pathological mitochondria, it may be beneficial for the subject [31-35]. In the present study, structural improvements, such as orientation of myocardial fibers, suppression of CM hypertrophy, and suppression of fibrosis (remodeling suppression) were observed. These histological changes could be induced by cytokines from the transplanted cells. Moreover, considering the downward trend of ejection fraction eight weeks post transplantation, in the aGA group, mitochondrial transfer could be considered to contribute not only to short-term, but also long-term improvements in CM function.

Various transfer modes have been proposed for mitochondrial transfer, including involvement of microvesicles, tunneling nanotubes, cell fusion, and potential cell surface gap junction proteins [36-38]. In vitro, danger-associated molecular pattern proteins, mtDNA, $\mathrm{Ca}^{2+}, \mathrm{CD} 38$ signaling, $\mathrm{NAD}^{+}$, and NADH, released from damaged cells, act to trigger signals and actively promote mitochondrial transfer from donor cells via modes shown in Graphical Abstract [39]. Additionally, evaluation of the direct myocardial transplantation of isolated mitochondria suggest a mechanism whereby cardiomyocytes directly internalize mitochondria through the cell membrane. These processes are considered to be non-specific and reflect passive transfer [40-43]. Herein, mitochondrial transfer was promoted by hypoxic stimulation in vitro, and mitochondrial transfer from the myocardium to hADSCs was prevented by a connexin blocker in vivo. Therefore, ischemic myocardial signaling may cause cell-cell communication via gap junctions, after which mitochondrial transfer occurs.

In this study, however, the transfer of mitochondrial DNA alone, migration of transplanted cells into the myocardium along with subsequent fusion with recipient cells, and mitochondrial transfer via 
microvesicles could not be excluded. The presence or absence of a signal from the recipient $\mathrm{CM}$ to the donor cell (to assess whether mitochondrial transfer is active or passive) was not determined, and any potential signal was not specified. Moreover, the amount and period of retention of transferred mitochondria, the mechanisms underlying subsequent increase in intramyocardial ATP, and the short- and long-term improvement of cardiac function remain to be fully elucidated. Nevertheless, our current findings support the hypothesis of cell transplantation delivering living mitochondria directly to myocardial cells, along with cytokines that promote angiogenesis, inducing protective effects in the myocardium [8]. Taken together, our results suggest that ADSC-to-CM mitochondrial transfer occurs both in vitro and in vivo, thereby contributing to the recovery of early cardiac function, following ADSC transplantation in ischemic cardiomyopathy. Thus, enhancement of the mitochondrial transfer mechanisms may be expected to lead to enhanced efficacy of cell therapy.

\section{Methods}

Experimental protocols were approved by the Ethics Review Committee for Animal Experimentation of Osaka University Graduate School of Medicine, Japan. Animal care was conducted humanely in compliance with the Principles of Laboratory Animal Care formulated by the National Society for Medical Research, and the Guide for the Care and Use of Laboratory Animals prepared by the Institute of Animal Resources and published by the National Institutes of Health (Eighth Edition, revised 2011).

\section{Assessment of in vivo mitochondrial transfer}

Transplantation of adipose tissue-derived stem cells (ADSCs) harboring stained mitochondria

A myocardial infarction model in F344/NJcl-rnu/rnu rats (CLEA Japan, Inc., Tokyo, Japan) was generated by permanent ligation of the left anterior descending artery (LAD). Two weeks following LAD ligation, human ADSCs (hADSCs; $1 \times 10^{6}$ cells; Lonza, Tokyo, Japan) were transplanted into the infarct area using fibrinogen and thrombin solution (CSL Behring, Tokyo, Japan). The composition of this graft is shown in Supplementary Table S1 online [17]. The hADSCs were pre-stained with MitoTracker Red (Thermo Fisher Scientific, Waltham, MA, USA) [18]. The rats were euthanized at appropriate time points following transplantation, and heart samples were collected (Fig. 6). Following formalin fixation, paraffin-embedded heart sections were stained with an antibody against phalloidin (Cat. \#A12379; Thermo Fisher Scientific) and counterstained with Hoechst 33342 (Dojindo Molecular Technologies, Inc., Kumamoto, Japan) (Supplementary material).

Observation of graft-heart boundaries with electron microscopy

Mitochondrial ultrastructure and spatial relationship between hADSCs and rat cardiomyocytes (rCMs) were assessed in tissue samples collected from areas including the boundary between heart and hADSC graft. Contrasted sections were imaged under an H-7500 transmission electron microscope (Hitachi, Tokyo, Japan) (Supplementary material). 
Myocardial infarction-induced adult male CAG/eGFP transgenic Sprague-Dawley (SD) rats (body weight, 200 - 250 g; Japan SLC, Inc., Shizuoka, Japan) were used as recipients of hADSC transplants. The hADSCs were transfected using CellLight ${ }^{\mathrm{TM}}$ Mitochondria-RFP BacMam 2.0 (Thermo Fisher Scientific) (Fig. 2A). Graft composition and transplantation was performed as explained above (Supplementary Table S1 online). The beating heart and the graft were stabilized using a custom-made suction device (Fig. 2B) [19]. Clear in vivo images were obtained by methods reported previously [20]. Observation was initiated immediately following transplantation and continued for maximum possible duration (Supplemental material).

\section{Detection of donor mitochondrial DNA in recipient rCMs}

Paraffin-embedded tissues from the heart and hADSC graft were harvested three days post transplantation. Tissue sections were stained with hematoxylin and eosin, and residual rCMs around the fibrotic tissue and just beneath the hADSC graft were collected using a laser-equipped microscope (Leica LMD7000). DNA was isolated, and a human mitochondrial DNA fragment was amplified using a customized primer pair specific for human mitochondria (Eurofins Genomics, Louisville, KY, USA) (Supplementary Table S2 online) [21-23]. PCR conditions and reaction mixtures are listed in Supplementary Tables S3 and S4 online. The PCR products were purified and used as a template for sequencing and further analysis (Supplementary material online).

\section{In vitro observation of mitochondrial transfer and its influence on rCM metabolism}

\section{Observation of rCM and hADSC co-culture}

Commercially available neonatal rCMs (RCM-561; QBM Cell Science, Ontario, Canada) were stained with MitoTracker Green (Thermo Fisher Scientific), seeded on 4-well glass slide chamber, and cultured under normoxic conditions for $3-4 \mathrm{~h}$. The same count of hADSCs, cultured under normoxic condition and stained with MitoTracker Red (Thermo Fisher Scientific), was transferred to the rCM culture, and the two cell populations were co-cultured for $1 \mathrm{~h}$ before observation (Fig. 3A) under hypoxic conditions $\left(1 \% \mathrm{O}_{2}\right)$, with or without $10 \mu \mathrm{M}$ aGA (Sigma-Aldrich, St. Louis, MO, USA), for $24 \mathrm{~h}$. Then, co-cultured cells were fixed and stained with anti-cardiac actinin and anti-human mitochondria antibodies, and three-dimensional images were constructed (Supplementary material).

\section{Measurement of oxygen consumption rate}

Oxygen consumption rate (OCR) in living cells was measured using a Seahorse XF24 Extracellular Flux Analyzer (Agilent Technologies, Santa Clara, CA, USA) according to the manufacturer's protocol. rCMs (2 $\times 10^{4}$ cells/well) were cultured on 96-well Agilent Seahorse XF cell culture microplates under normoxic conditions for $48 \mathrm{~h}$, after which the same number of hADSCs were added to each well and cultured with 
or without aGA, under normoxic conditions, for $24 \mathrm{~h}$ before analysis. The detailed culture conditions for each group are listed in Supplementary Table S5 online [24].

Quantification of cytokines from the hADSC graft

hADSC grafts were cultured under normoxic or hypoxic $\left(1 \% \mathrm{O}_{2}\right)$ conditions, and culture supernatant was collected after 24,48 , and $72 \mathrm{~h}$. The secretion of hepatocyte growth factor (HGF), vascular endothelial growth factor (VEGF), IL-6, and IL-10, which has been reported to be associated with functional improvement, was measured by performing enzyme-linked immunosorbent assays (R\&D Systems, Minneapolis, MN, USA).

Isolation and motion analysis of rCMs with/without hADSC supernatant

The 7-8-week-old male Crl:CD (SD) rats were anesthetized by inhalation of isoflurane, and heart was harvested. The heart was connected to a Langendorff circulation circuit through aortic ligation to the cannula (Fig. 3G). The perfusion buffer (Tyrode's solution, Supplementary Table S6 online) containing 300 units/mL collagenase type II (Gibco; Thermo Fisher Scientific) was perfused into the coronary arteries. The heart was homogenized on a petri dish, and cardiac cells were pipetted and transferred to a 96-well plate (Fig. $3 \mathrm{H}-\mathrm{I})$. The cardiomyocytes were stimulated with either $1 \mu \mathrm{M}$ isoprenaline, $400 \mu \mathrm{M}$ propranolol, or hADSC culture supernatant (Fig. $3 \mathrm{~J}$ ). Motion of rCMs was observed and analyzed using the SI8000 Cell Motion Imaging System (Sony, Tokyo, Japan) (Supplementary material online) [25-27].

\section{Evaluation of influence of mitochondrial transfer on cardiac function andmetabolism}

\section{Graft survival following transplantation}

Luciferase-transduced hADSCs were transplanted into the nude rat myocardial infarction model in the same manner as described above (Fig. 4A). Luminescence intensity of the grafts was measured at the body surface. For histological analysis, hearts with residual graft samples were collected at days 1, 3, 7, and 14, and paraffin-embedded samples were stained using an antibody specific to human mitochondria (Cat. \#MAB1273; Merck Millipore, Burlington, MA, USA) (Supplementary material).

Assessment of cardiac function

Following the injections with hADSCs $\left(1 \times 10^{5}\right.$ cells/body (low-dose group), $5 \times 10^{5}$ cells/body (mediumdose group), $1 \times 10^{6}$ cells/body (high-dose group), and $5 \times 10^{6}$ cells/body (max-dose group)), cardiac function was assessed using an echocardiography system equipped with a $12 \mathrm{MHz}$ transducer (SONOS 7500; Philips, Amsterdam, Netherlands). Heart samples were collected on days 3, 7, 14, and 56 following transplantation (Supplementary material).

Two weeks after inducing myocardial infarction, either hADSCs (A group, $n=20$ ), hADSCs combined with aGA (final concentration, $10 \mu \mathrm{M} ; \mathrm{A}+$ aGA group, $\mathrm{n}=10$ ), or aGA alone (final concentration, $10 \mu \mathrm{M}$; aGA group, $n=4$ ) were transplanted. For the control group, sham operation performed (fibrinogen and 
thrombin solution only; $\mathrm{C}$ group, $\mathrm{n}=10$ ). Cardiac function was examined using echocardiography. Heart samples were collected on days $3,7,14$, and 56 following transplantation (Supplementary material).

Histological analysis of the heart

Heart samples were obtained from each group 8 weeks following surgery. To assess rCM diameter and fibrosis, heart sections were stained with periodic acid-Schiff and Picro Sirius red, respectively. The sections were also stained with anti-von Willebrand factor antibody, and capillary density was calculated in the peri-infarct area. For morphological evaluation of mitochondria in the heart, electron microscopy was performed as described above.

Quantification of intramyocardial ATP and mitochondrial DNA

ATP content in the heart samples was assessed using a colorimetric ATP assay kit (ab83355; Abcam, Cambridge, UK). To quantify human and rat mitochondrial DNA in the recipient heart, we performed quantitative PCR. Total DNA was isolated from the infarct region and remote areas, and PCR was performed with SYBR Green (Thermo Fisher Scientific) using specific primer pairs (Supplementary Table S1 online).

\section{Statistical analysis}

All values are expressed as means \pm SEM. Statistical analyses were performed using JMP Pro 14 software (SAS Institute Inc., Cary, NC, USA). Comparison across multiple groups with normal distribution was performed using one-way analysis of variance (ANOVA), followed by Tukey's multiple comparisons test. Comparison between two groups was performed using an unpaired or paired two-tailed Student's $t$ test or Mann-Whitney Utest, as appropriate. Comparison across multiple groups with non-normal distribution was performed using Kruskal-Wallis tests followed by Dunn's multiple comparisons tests. $p$ $<0.05$ were considered statistically significant.

\section{Declarations}

\section{Data Availability}

\section{Acknowledgments}

The authors are grateful to Dr. Hiroko Iseoka, Dr. Fumiya Oohashi, and Dr. Shohei Yoshida for their invaluable technical assistance.

\section{Author Contributions}

D.M. participated in research design and performance, analyzed data, and wrote the article.

M.O., K. K., H. K., and H. N. performed research experiments and analyzed data. 
1. Kawamura., D. Y., H. H., T. U., K. T., T. Kuratani, and T. T. reviewed the manuscript.

2. M. and Y. S. participated in research design and reviewed all data and the manuscript.

All authors have performed the following role: Drafting the work or revising it critically for important intellectual content; final approval of the version to be published; agreement to be accountable for all aspects of the work in ensuring that questions related to the accuracy or integrity of any part of the work are appropriately investigated and resolved.

\section{Sources of Funding}

This study was supported by ROHTO Pharmaceutical Co., Ltd.

\section{Competing interests}

Dr. Sawa serves as an advisor for the sponsor (ROHTO Pharmaceutical Co., Ltd.). Dr. Sawa and Dr. Miyagawa received remuneration for a lecture from the sponsor. Ms. Oota, Ms. Kawai, Mr. Kurata, and Mr. Nishida are employees of the sponsor. The other authors declare no competing financial interest. The other authors declare no competing financial interest. The sponsor had no control over the interpretation, writing, or publication of this study. The terms of this arrangement have been reviewed and approved by the Osaka University in accordance with its policy on objectivity in research.

\section{References}

1. Cahill, T.J., Choudhury, R.P. \& Riley, P.R. Heart regeneration and repair after myocardial infarction: translational opportunities for novel therapeutics. Rev. Drug Discov. 16, 699-717 (2017).

2. Kloner, R.A. et al. New and revisited approaches to preserving the reperfused myocardium. Rev. Cardiol. 14, 679-693 (2017).

3. Lopez-Crisosto, C. et al. Sarcoplasmic reticulum-mitochondria communication in cardiovascular pathophysiology. Rev. Cardiol.14, 342-360 (2017).

4. Wu, R., Hu, X. \& Wang, J. Concise review: optimized strategies for stem cell-based therapy in myocardial repair: Clinical translatability and potential limitation. Stem Cel/s36, 482-500 (2018).

5. Rustom, A. et al. Nanotubular highways for intercellular organelle transport. Science303, 1007-1010 (2004).

6. Islam, M.N. et al. Mitochondrial transfer from bone-marrow-derived stromal cells to pulmonary alveoli protects against acute lung injury. Med. 18, 759-765 (2012).

7. Jackson, M.V. et al. (2016). Mitochondrial transfer via tunneling nanotubes is an important mechanism by which mesenchymal stem cells enhance macrophage phagocytosis in the in vitro and in vivo models of ARDS. Stem Cells. 34, 2210-2223 (2016).

8. Brown, D.A. et al. Expert consensus document: Mitochondrial function as a therapeutic target in heart failure. Rev. Cardiol. 14, 238-250 (2017). 
9. Paliwal, S. et al. Regenerative abilities of mesenchymal stem cells through mitochondrial transfer. Biomed. Sci. 25, 31 (2018).

10. Koyanagi, M. et al. Cell-to-cell connection of endothelial progenitor cells with cardiac myocytes by nanotubes: a novel mechanism for cell fate changes. Res. 96, 1039-1041 (2005).

11. Plotnikov, E.Y. et al. (2008). Cell-to-cell cross-talk between mesenchymal stem cells and cardiomyocytes in co-culture. Cell Mol. Med. 12, 1622-1631 (2008).

12. Pacak, C.A. et al. Actin-dependent mitochondrial internalization in cardiomyocytes: evidence for rescue of mitochondrial function. Open.4, 622-626 (2015).

13. Han, H. et al. (2016). Bone marrow-derived mesenchymal stem cells rescue injured H9c2 cells via transferring intact mitochondria through tunneling nanotubes in an in vitro simulated ischemia/reperfusion model. Med. Rep. 13, 1517-1524 (2016).

14. Li, J., King, N.C. \& Sinoway, L.I. ATP concentrations and muscle tension increase linearly with muscle contraction. Appl. Physiol. 95, 577-583 (2003).

15. Eisner, V. et al. (2017). Mitochondrial fusion dynamics is robust in the heart and depends on calcium oscillations and contractile activity. Natl. Acad. Sci. U. S. A.114, E859-E868 (2017).

16. Wang, K. et al. Mitochondria regulate cardiac contraction through ATP-dependent and independent mechanisms. Free Radic. Res. 52, 1256-1265 (2018).

17. Mori, D. et al. Pioglitazone strengthen therapeutic effect of adipose-derived regenerative cells against ischemic cardiomyopathy through enhanced expression of adiponectin and modulation of macrophage phenotype. Diabetol.18, 39 (2019).

18. Berridge, M.V. et al. Mitochondrial transfer between cells: methodological constraints in cell culture and animal models. Biochem. 552, 75-80 (2018).

19. Matsuura, R. et al. (2018). Intravital imaging with two-photon microscopy reveals cellular dynamics in the ischeamia-reperfused rat heart. Rep. 8, 15991 (2018).

20. Vinegoni, C. et al. (2015). Imaging the beating heart in the mouse using intravital microscopy techniques. Protoc. 10, 1802-1819 (2015).

21. Rooney, J.P. et al. PCR based determination of mitochondrial DNA copy number in multiple species. Methods Mol. Biol. 1241, 23-38 (2015).

22. Ayala-Torres, S. et al. Analysis of gene-specific DNA damage and repair using quantitative polymerase chain reaction. Methods. 22, 135-147 (2000).

23. Branda, R.F. et al. Dietary modulation of mitochondrial DNA deletions and copy number after chemotherapy in rats. Res. 501, 29-36 (2002).

24. Hawat, G. et al. Connexin 43 mimetic peptide Gap26 confers protection to intact heart against myocardial ischemia injury. Pflugers Arch.460, 583-592 (2010).

25. Louch, W.E., Sheehan, K.A. \& Wolska, B.M. Methods in cardiomyocyte isolation, culture, and gene transfer. Mol. Cell Cardiol. 51, 288-298 (2011). 
26. Li, D.X. et al. Isolation and culture of adult mouse cardiomyocytes for cell signaling and in vitro cardiac hypertrophy. Vis. Exp. 87, 51357 (2014).

27. Iseoka, H. et al. Pivotal role of non-cardiomyocytes in electromechanical and therapeutic potential of induced pluripotent stem cell-derived engineered cardiac tissue. Tissue Eng. A.24, 287-300 (2018).

28. Kitani, T.Kami, D., Matoba, S. \& Gojo, S. Internalization of isolated functional mitochondria: involvement of macropinocytosis. Cell Mol. Med. 18, 1694-1703 (2014).

29. Gherghiceanu, M. \& Popescu, L.M. Cardiac telocytes-their junctions and functional implications. Cell Tissue Res. 348, 265-279 (2012).

30. Kitani, T. et al. (2014). Internalization of isolated functional mitochondria: involvement of macropinocytosis. Cell Mol. Med. 18, 1694-1703 (2014).

31. Meyers, D.E., Basha, H.I. \& Koenig, M.K. Mitochondrial cardiomyopathy: pathophysiology, diagnosis, and management. Tex Heart Inst. J.40, 385-394 (2013).

32. Hallas, T. et al. Investigating the cardiac pathology of SCO2-mediated hypertrophic cardiomyopathy using patients induced pluripotent stem cell-derived cardiomyocytes. Cell Mol. Med. 22, 913-925 (2018).

33. Joubert, M. et al. Diabetes-related cardiomyopathy: the sweet story of glucose overload from epidemiology to cellular pathways. Diabetes Metab.45, 238-247 (2019).

34. Stanzani, G., Duchen, M.R. \& Singer, M. The role of mitochondria in sepsis-induced cardiomyopathy. Biophys.Acta Mol. Basis Dis. 1865, $759-73$ (2019).

35. Viola, H.M. \& Hool, L.C. Impaired calcium handling and mitochondrial metabolic dysfunction as early markers of hypertrophic cardiomyopathy. Biochem. Biophys.665, 166-174 (2019).

36. Tan, A.S. et al. Mitochondrial genome acquisition restores respiratory function and tumorigenic potential of cancer cells without mitochondrial DNA. Cell Metab. 21, 81-94 (2015).

37. Denuc, A. et al. New protein-protein interactions of mitochondrial connexin 43 in mouse heart. Cell Mol. Med.20, 794-803 (2016).

38. Torralba, D., Baixauli, F. \& Sánchez-Madrid, F. Mitochondria know no boundaries: mechanisms and functions of intercellular mitochondrial transfer. Cell Dev. Biol.4, 107 (2016).

39. Paliwal, S. et al. Human tissue-specific MSCs demonstrate differential mitochondria transfer abilities that may determine their regenerative abilities. Stem Cell Res. Ther. 9, 298 (2018).

40. Cowan, D.B. et al. (2017). Transit and integration of extracellular mitochondria in human heart cells. Rep. 7, 17450 (2017).

41. Kaza, A.K. et al. Myocardial rescue with autologous mitochondrial transplantation in a porcine model of ischemia/reperfusion. Thorac. Cardiovasc. Surg.153, 934-943 (2017).

42. Emani, S.M. \& McCully, J.D. Mitochondrial transplantation: applications for pediatric patients with congenital heart disease. Pediatr.7, 169-175 (2018).

43. Wang, J. et al. Stem cell-derived mitochondria transplantation: a novel strategy and the challenges for the treatment of tissue injury. Stem Cell Res. Ther. 9, 106 (2018). 


\section{Figures}
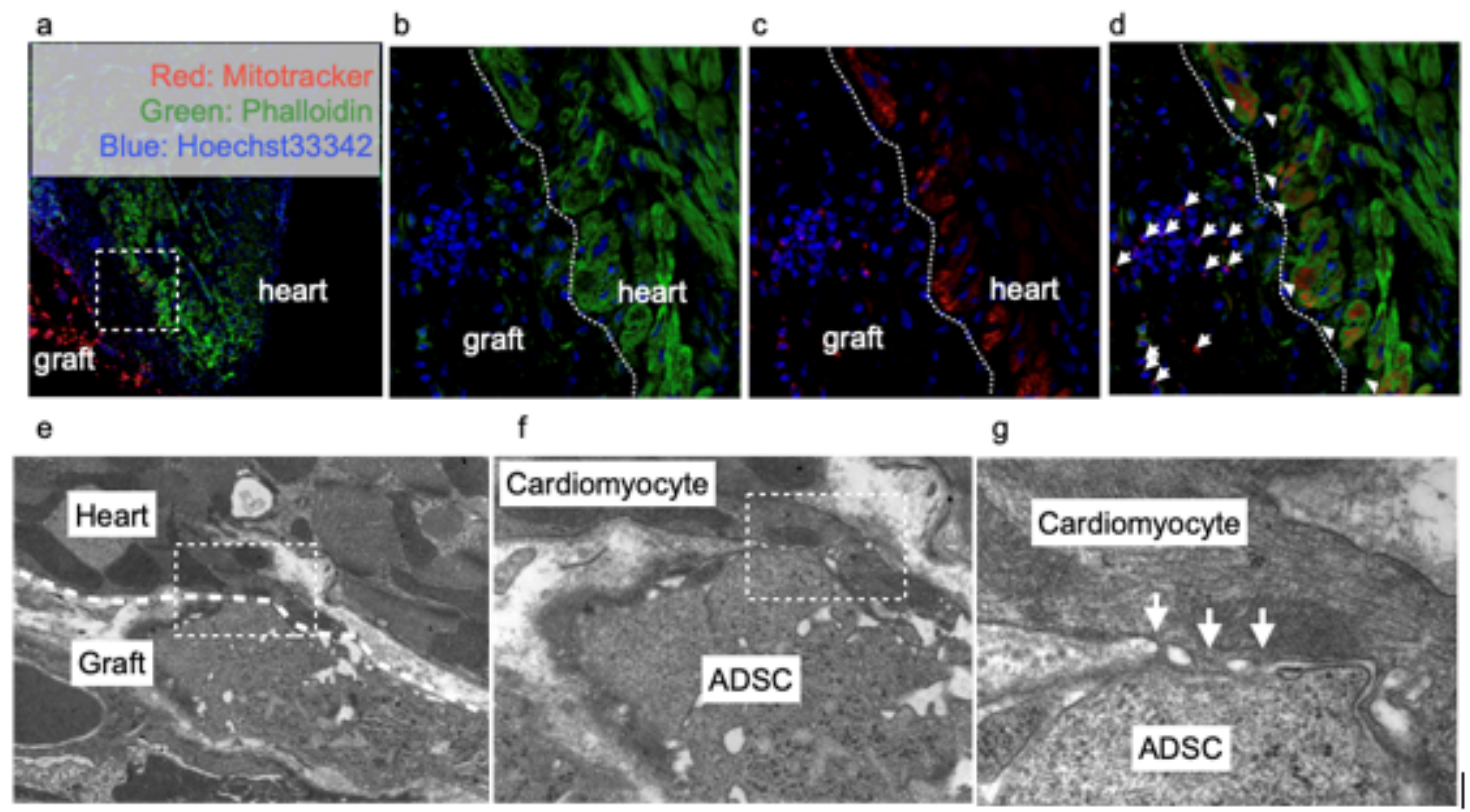

f $g$

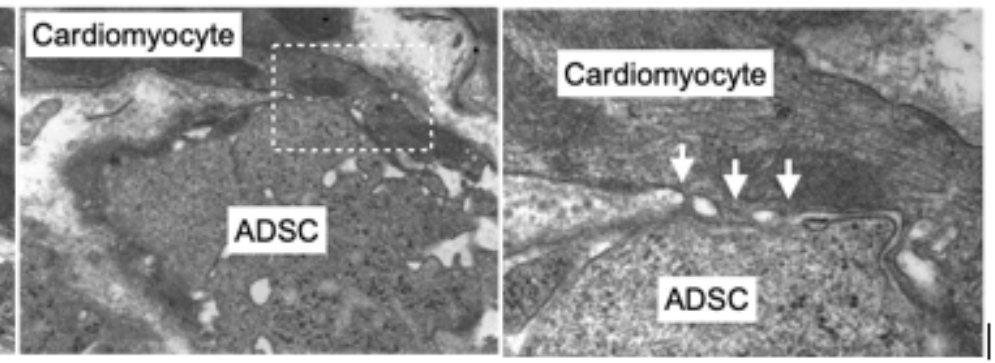

$\mathrm{h}$

i

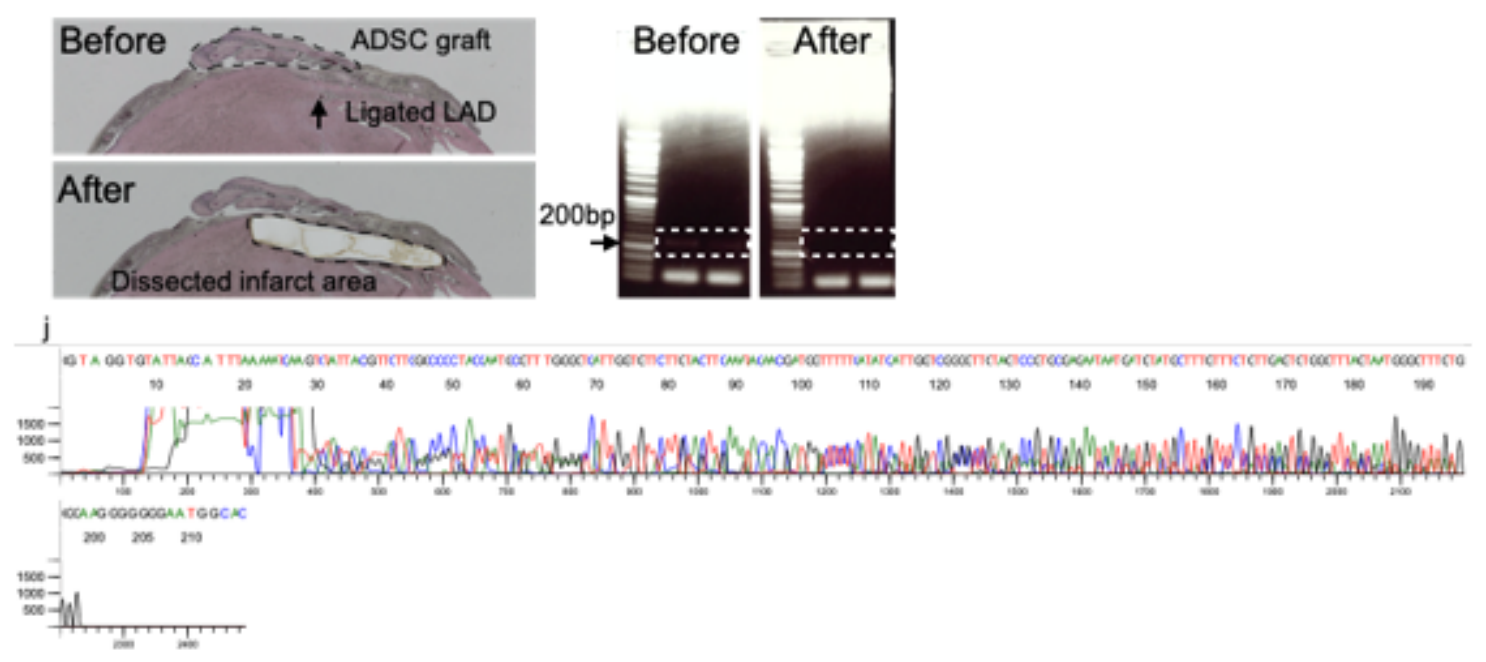

\section{Figure 1}

Evaluation of mitochondrial transfer in vivo. (A) Three days following transplantation, hADSC graftderived mitochondria were observed in recipient $\mathrm{rCMs}$; the epicardium and mitochondria in the hADSC graft were also retained. This transfer phenomenon was observed only in rats with myocardial infarction. $(B, C)$ Individual staining for rat actin and human mitochondria are shown in the dashed line box in $A$. (D) Magnified picture of the dashed line box in A. Arrow, mitochondria in ADSC; arrowhead, mitochondria in $\mathrm{CM}$; red, mitochondria of $\mathrm{ADSC}$; green, actin in $\mathrm{rCM}$; blue, nucleus. (E) Electron microscopy image of the graft and heart boundary. (F) Magnified picture of the dashed rectangle in E. (G) Magnified picture of the dashed rectangle in F. Arrows indicate pseudopod-like structures or fusion. $(\mathrm{H})$ Heart sample used for sequencing the donor mitochondrial DNA fragment. The samples for sequencing were micro-dissected and obtained from the part enclosed by dashed line. (I) Target amplicons (rectangle) were duplicate 
electrophoresed on a single gel. A band was confirmed at 200-250 bp. (J) Gene sequence homology to human MT-ND6. MT-ND6, mitochondrially encoded NADH-ubiquinone oxidoreductase core subunit 6.

a

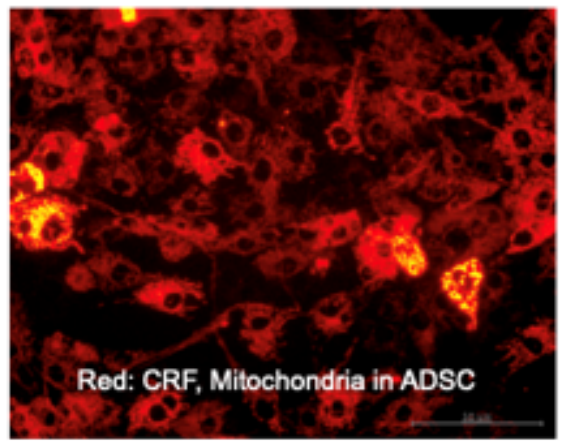

c

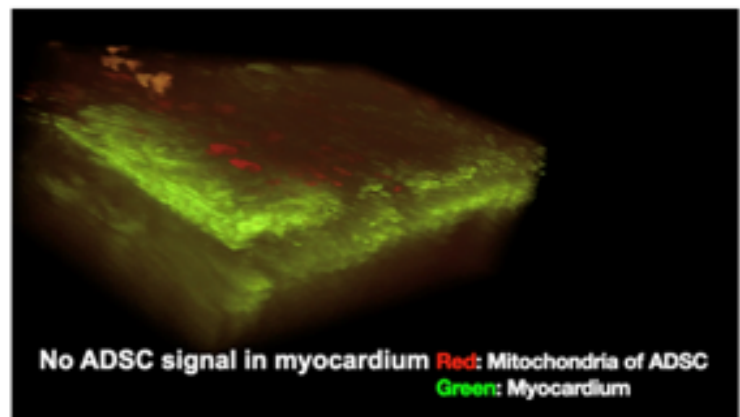

d
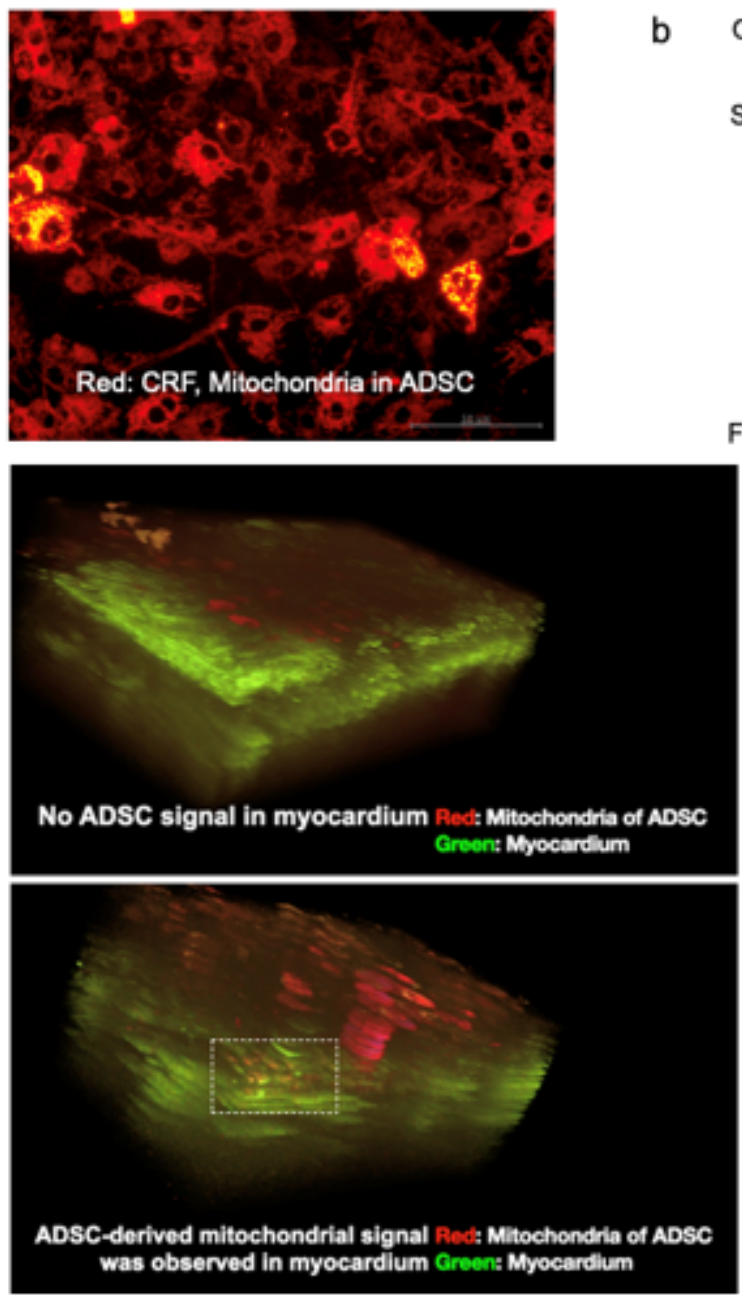

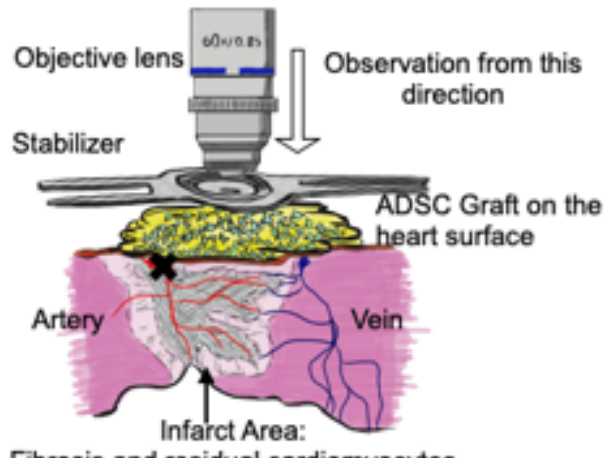

Fibrosis and residual cardiomyocytes

e

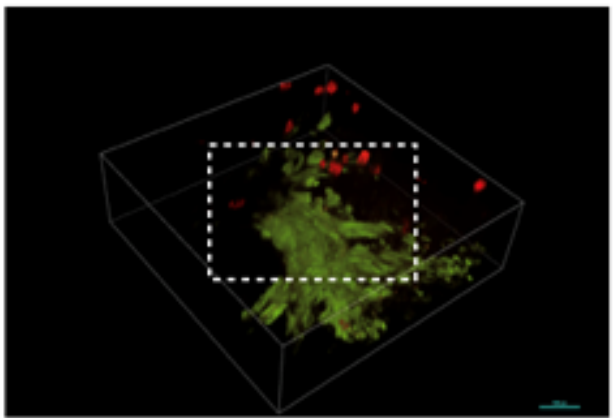

f

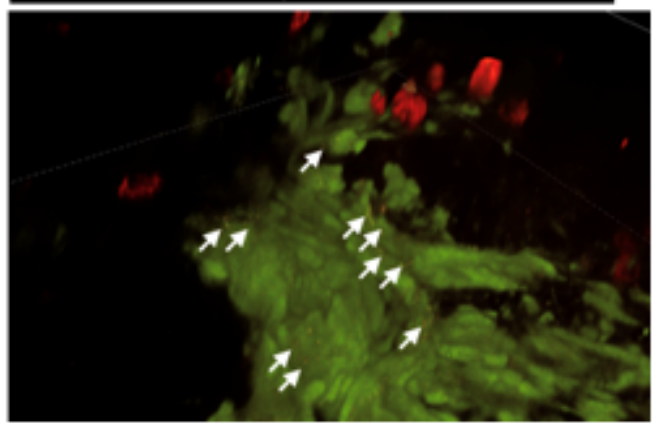

\section{Figure 2}

Intravital imaging of mitochondrial transfer. (A) hADSCs transfected with CellLight to express red fluorescent protein in the mitochondria. (B) Schematic illustration of intravital imaging. The residual rCMs in the infarct area were observed through the stabilizer and graft. (C) Spatial relationship between the graft and heart at the beginning of observation. (D) RFP signal in the rCMs just beneath the pericardium (rectangle) six hours after grafting. (E) Representative image of residual rCMs in the infarct area. Arrows indicate transferred granular mitochondria. $(F)$ Magnified picture of the dashed rectangle in $E$. 
a

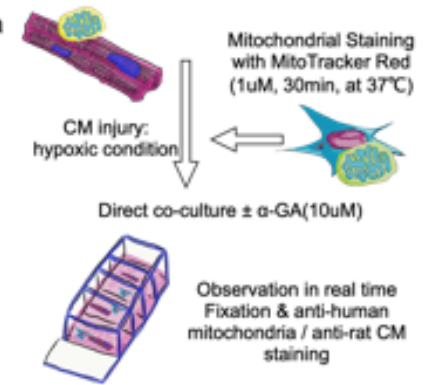

e

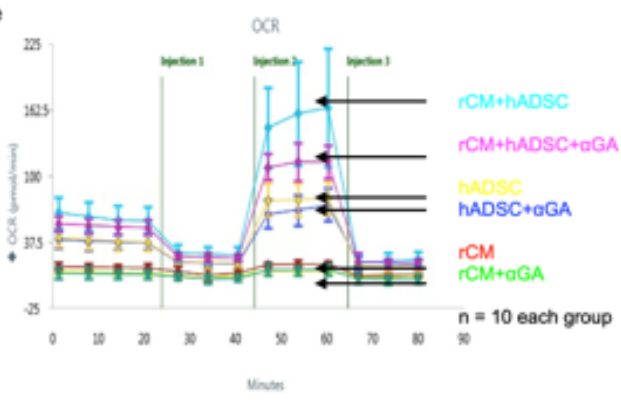

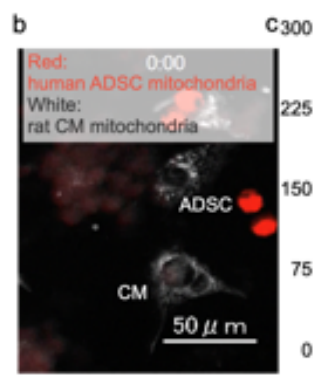

f

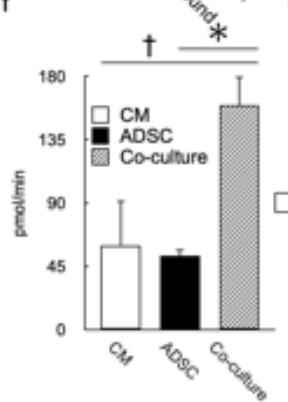

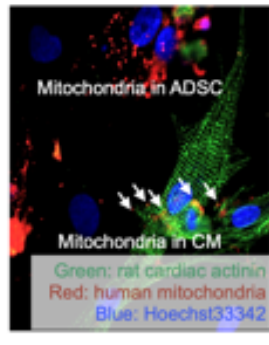

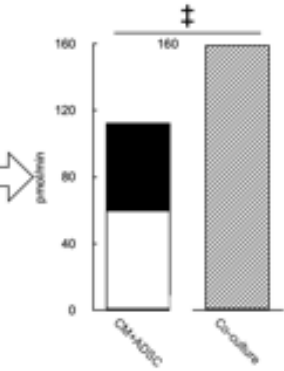

$* p<0.05$ vs. ADSC $t p<0.05$ vs. CM ₹p<0.05 g
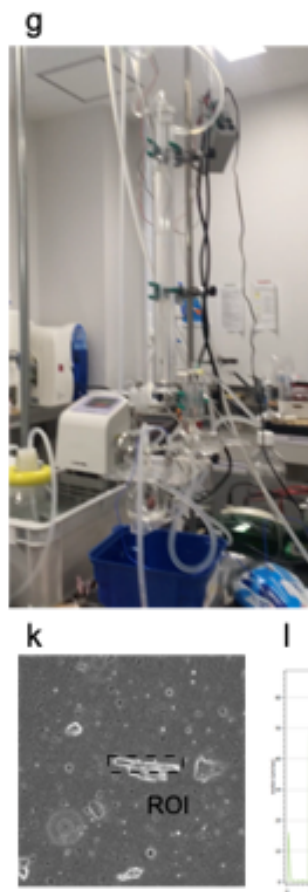

I h

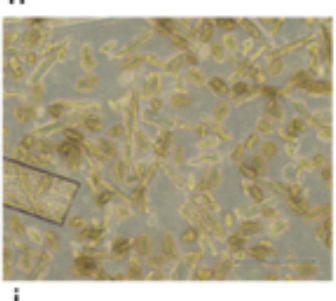

i



j
Add culture supernatant of ADSC

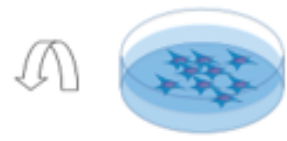

The adult rat myocardium was isolated using the Langendorff circuit.

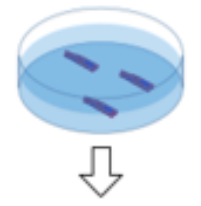

Observe with motion analyzer

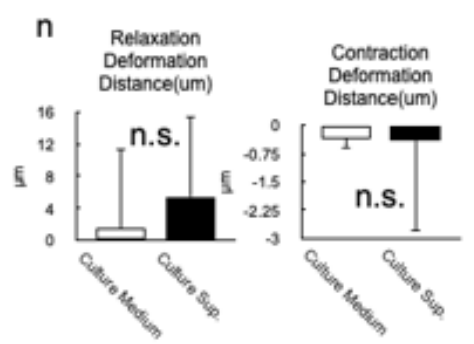

\section{Figure 3}

Co-culture experiment of rCMs and hADSCs. (A) rCMs were cultured on chamber slides under normoxic conditions for $3-4 \mathrm{~h}$, and pre-stained hADSCs were added thereafter. (B) Real-time continuous observations over $24 \mathrm{~h}$ with or without aGA under hypoxic condition. (C) Mitochondrial luminescence intensity in rCMs and culture medium. (D) Fixed co-culture samples showing the transferred granular mitochondria in rCMs; $n=4$ samples per group. ${ }^{*} p<0.05$ by t-test. $(E, F)$ Effect of aGA on OCR of rCMs 
and hADSCs cultured independently or co-cultured; $n=10$ per group. ${ }^{\star} p<0.05, t p<0.05, \neq p<0.05$ by ANOVA. (G) Illustration of the isolation system of adult rCMs. $(\mathrm{H}, \mathrm{I})$ Representative image of harvested rCMs. $(J)$ Sequence of this experiment: Culture supernatant of hADSCs was collected and added to the freshly isolated adult rCMs for motion analysis. (K) In motion analysis, the region of interest was set independently for each adult rCM. (L, M) Representative velocity wave forms. (N) Effect of hADSC culture supernatant on rCM contractions.

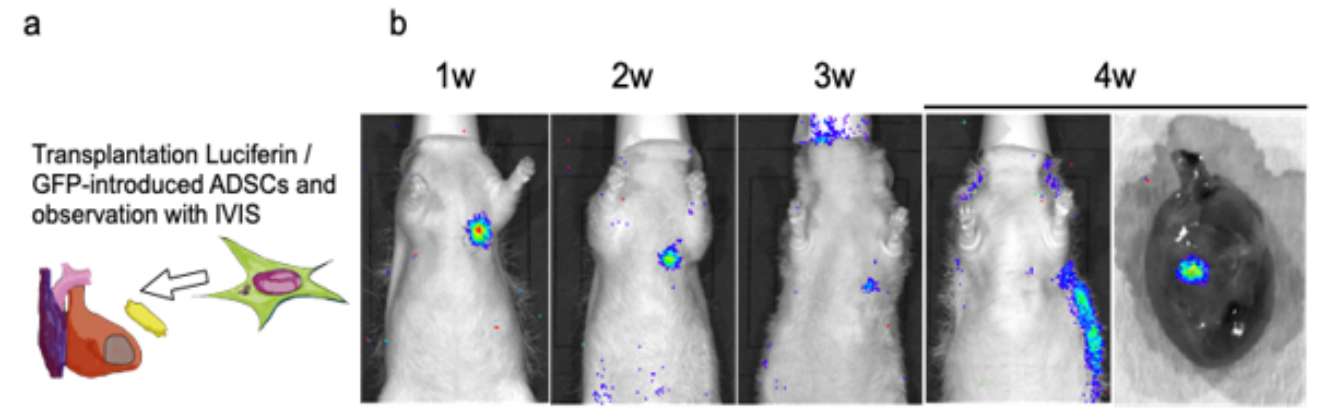

\section{c}

d
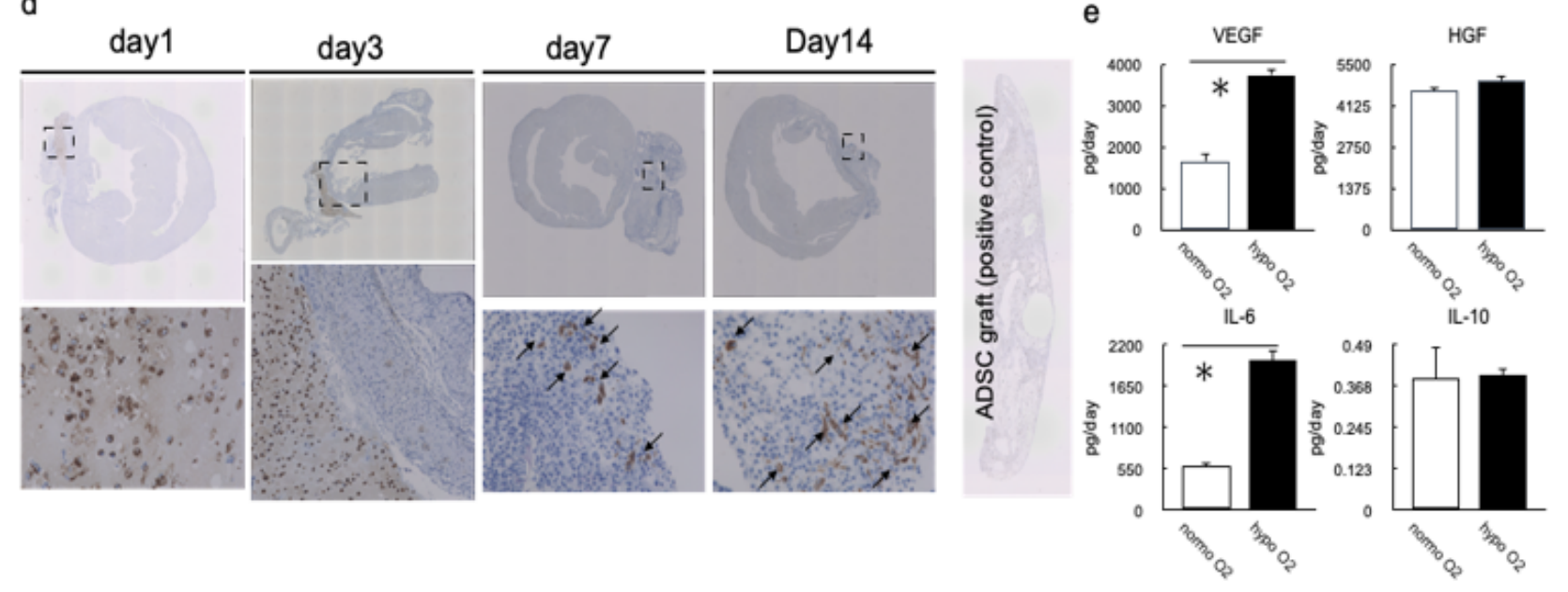

$f$
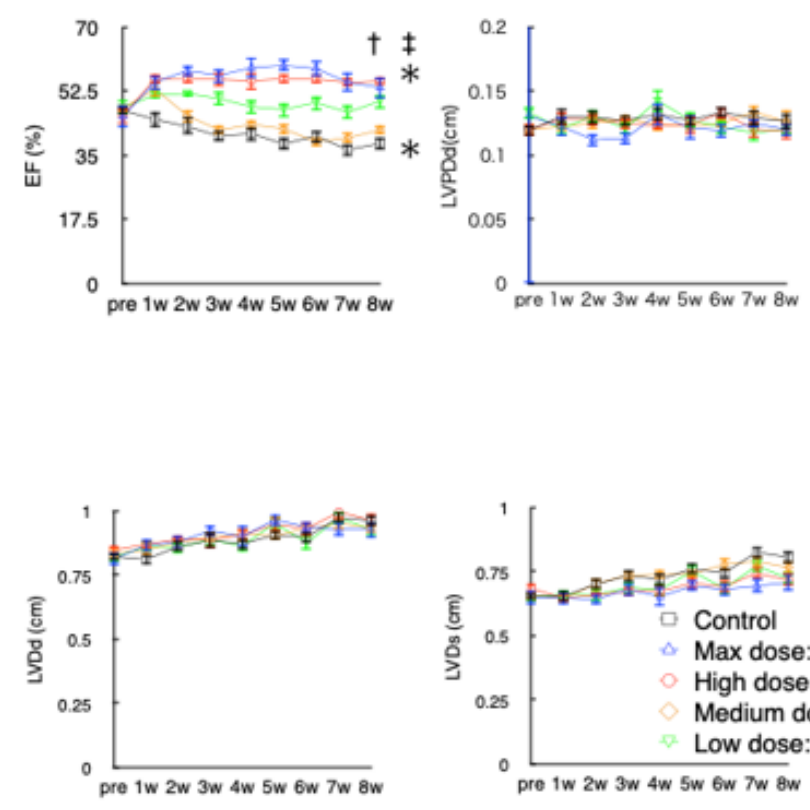

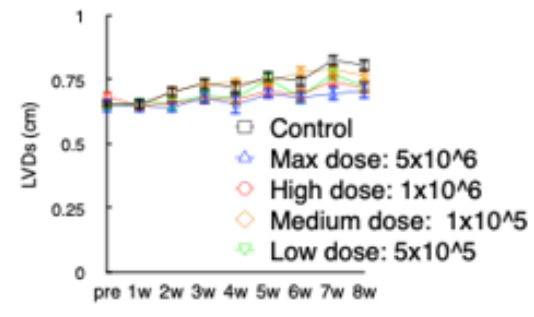

g




hADSC transplantation into a rat model of ischemic cardiomyopathy. (A) For evaluation of hADSC survival and engraftment, luciferin-transduced hADSCs were implanted on the surface of the heart. (B) Representative image of the luciferin signal from hADSCs. (C) Luciferin signal intensity showing the survival of hADSCs in the graft over time. (D) Representative images of hADSC grafts with stained antimitochondria antibodyu. Lower images are magnifications of the dashed boxes in the upper images. Image on the far right shows the whole graft. (E) Cytokine secretion from hADSC grafts measured using ELISA; $n=3$ samples per group. ${ }^{*} p<0.05$ by t-test. $(F)$ Cardiac function and dimension following transplantation; $\mathrm{n}=8$ per group. ${ }^{*} \mathrm{p}<0.05, \mathrm{t} p<0.05, \neq \mathrm{p}<0.05$ by ANOVA. (G) Long-term and short-term cardiac function in selected groups after hADSC transplantation; $\mathrm{n}=8$ rats per group. ${ }^{\star} \mathrm{p}<0.05 \mathrm{C}, \mathrm{tp}<$ $0.05 \neq p<0.05,9 p<0.05$ by ANOVA. 


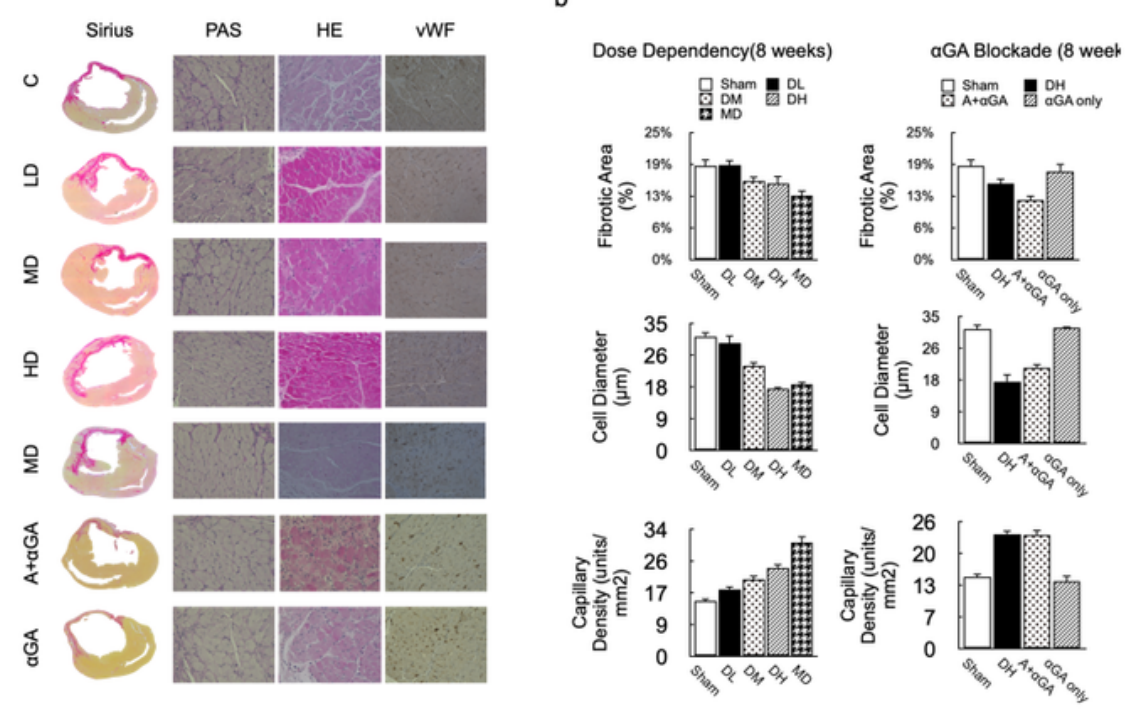

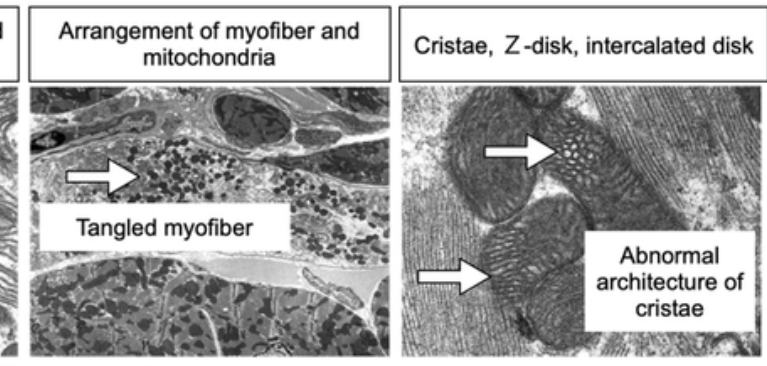
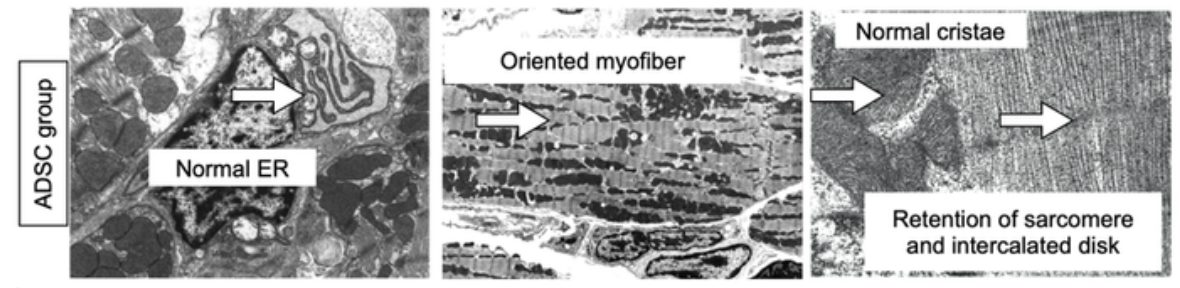

d
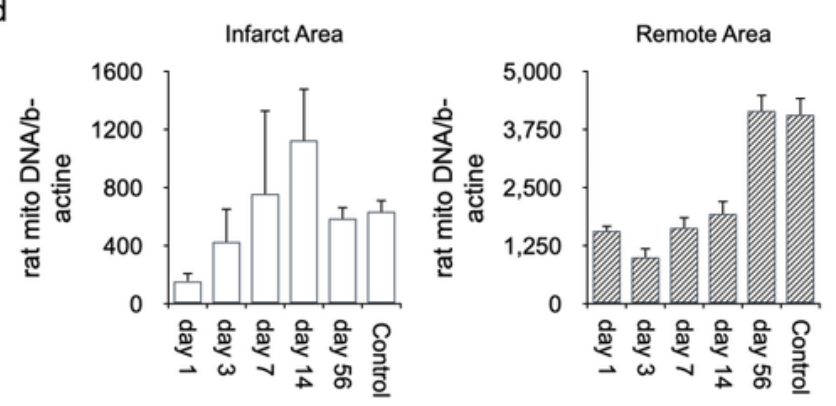

e

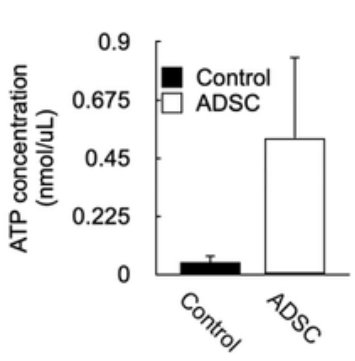

\section{Figure 5}

Histological analysis of the heart. (A) Representative whole microscopy images of Sirius red staining, periodic acid-Schiff staining, and immunostaining using an anti-von Willebrand factor antibody in each group; $n=8-10$ per group, scale bar $=50 \mu \mathrm{m}$. (B) Fibrotic area, capillary density, and rCM diameter in each group; $\mathrm{n}=8$ samples for each group; ${ }^{*} \mathrm{p}<0.05,+\mathrm{p}<0.05$ by ANOVA. (C) Morphology of mitochondria in rCMs that were observed using an electron microscope 8 weeks following 
transplantation. (D) Intramyocardial mitochondrial DNA of the recipient was quantified for each region: infarct and remote areas; $n=4$ samples for each group. ${ }^{*} p<0.05$ by ANOVA. (E) Intramyocardial ATP concentration one day following transplantation; $\mathrm{n}=3$ samples per group; ${ }^{*} \mathrm{p}<0.05$ by $\mathrm{t}$-test.


LEW rat: 7 weeks old ô, $200-250 \mathrm{~g}$

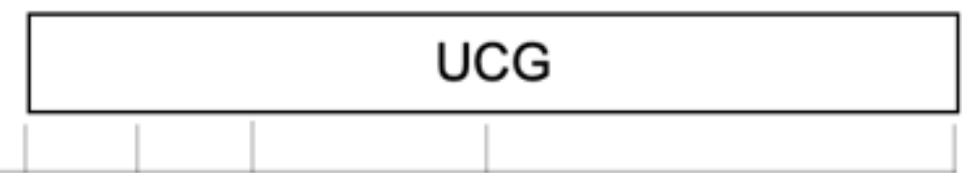

$-2 w$

Myocardial infarction

\begin{tabular}{|c|}
0 day3 $1 w \quad 2 w$ \\
$\begin{array}{c}\text { Histological and Biochemical analysis } \\
\text { of the heart sample }\end{array}$ \\
\hline
\end{tabular}

\section{Figure 6}

Study protocol. Myocardial infarction was induced in 7-8-week-old male rats two weeks prior to transplantation. During transplantation, hADSCs with or without pre-stained mitochondria were implanted using fibrin glue on the surface of the heart. Following transplantation, heart samples were obtained at scheduled time points for various analyses and cardiac function was evaluated using echocardiography. The hADSC grafts were analyzed in vitro.

\section{Supplementary Files}

This is a list of supplementary files associated with this preprint. Click to download.

- MTGraphicalaAbstract20201027.docx

- SupplementalMaterialforSR20201116.pdf 